\title{
Evaluation on Big Data Industry Development Level Based on Hesitant Fuzzy Linguistic TOPSIS Method
}

\author{
Lujing Pang ${ }^{\mathrm{a}, \mathrm{b}}$, Mu Zhang ${ }^{\mathrm{a}, \mathrm{b}}$ \\ a. School of Big Data Application and Economics, Guizhou University of Finance and Economics \\ b. Guizhou Institution for Technology Innovation \& Entrepreneurship Investment, Guizhou University of Finance and Economics \\ Guiyang 550025, China \\ rim_007@163.com

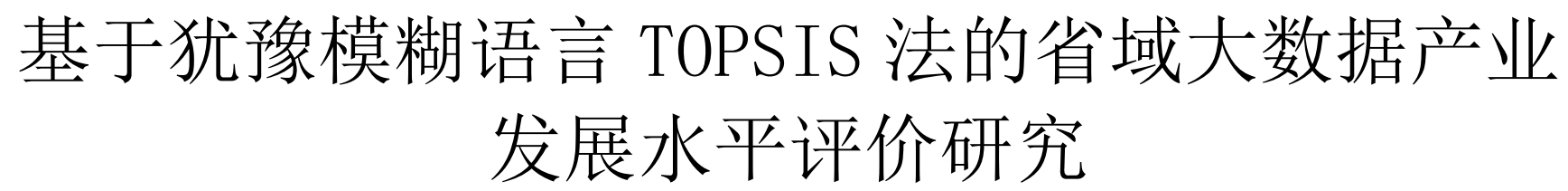

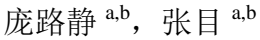 \\ a. 贵州财经大学大数据应用与经济学院 \\ $b$. 贵州财经大学贵州科技创新创业投资研究院,
}

贵阳 550025, 中国

rim_007@163.com

\begin{abstract}
Reasonable and effective evaluation of the development level of big data industry is conducive to promoting the development of big data in an all-round way and speeding up the construction of a data power. In view of the insufficient information of the existing index system and the lack of fuzziness and hesitation in evaluation methods, this paper introduces Baidu search index to construct the evaluation index system of provincial large data industry development level from five aspects: industrial scale, product type, investment attraction, infrastructure and industrial influence; and chooses 2013-2017. Based on the original data of annual indicators, this paper evaluates the development level of big data industry in China by using TOPSIS method. The empirical results show that the big data industry in China is in the stage of continuous development in terms of time, and that in terms of space, Guangdong and Jiangsu have relatively high level of development, ranking first and second in the country respectively. During the sample period, the national big data industry is in a state of continuous development, but there is an imbalance in regional development.
\end{abstract}

Keywords-Big Data Industry; Development Level of Big Data Industry; Baidu Index; Hesitative Fuzzy Linguistic Term Set; TOPSIS Method

摘要一合理、有效地评价大数据产业发展水平，有利于全 面推进大数据发展, 加快建设数据强国。文章针对已有指标 体系信息不充分和评价方法缺乏模糊性、犹豫性的局限，引 入百度搜索指数，从产业规模、产品类型、招商引资、基础 设施以产业影响力等五个方面构建省域大数据产业发展水平 评价指标体系; 并选取 2013-2017 年指标原始数据, 运用犹豫 模糊语言 TOPSIS 法对全国的大数据产业发展水平进行实证测 评。实证结果表明: 从时间上来看, 我国大数据产业处于总 体上不断发展的阶段; 从空间上来看, 广东和江苏的大数据 产业发展水平较高，分别位列全国第一和第二。样本期间
内, 全国大数据产业处于不断发展的状态, 但存在区域发展 不平衡问题。

\section{引言}

大数据的发展以及大数据技术的进步促使大数据产 业成为经济新增长点。大数据产业指以数据生产、采 集、存储、加工、分析、服务为主的相关经济活动, 包 括数据资源建设、大数据软硬件产品的开发、销售和租 赁活动，以及相关信息技术服务 ${ }^{[1]}$ 。2015 年国务院印发 《促进大数据发展行动纲要》, 全面推进大数据发展, 加快建设数据强国。2016 年工信部颁布了《大数据产业 发展规划（2016-2020 年）》，分析了我国大数据产业 面临的形势以及现阶段要完成的重点任务和重大工程。 大数据技术的发展, 使得大数据产业不断的进行发展和 突破, 大数据产业逐渐成为各个国家竞相布局的产业。 抢抓机遇, 推动大数据产业发展, 对提升政府治理能 力、优化民生公共服务、促进经济转型和创新发展有重 大意义。

理论上, 关于产业发展水平和能力水平评价的文献 比较丰富。张向先等 (1997) ${ }^{[2]}$ 运用代表集聚功能、孵 化功能、扩散功能、渗透功能、示范功能、波及功能的 指标对高技术产业开发区进行评价。唐中赋和顾培亮 (2003) [3] 高新技术产业发展水平的投人指标、产出指 标、效益指标及潜力指标进行评价。李琳和陈晓红 (2005) ${ }^{[4]}$ 构建技术创新能力、产业群集力、环境支撑 力、政府作用力、持续发展力五个一级指标体系, 运用 层次分析法确定权重, 并通过数据标准化进行综合评 价。张危宁等 (2006) ${ }^{[5]}$ 主要通过知识流动能力、技术 创新能力、创新环境、创新经济绩效四个主要方面来对 高技术产业集群创新绩效进行评价。陈伟（2010） ${ }^{[6] 从 ~}$ 技术价值、经济价值和社会效益三个维度,构建高技术产 
业 R\&D 绩效评价指标体系。陈红川（2010） ${ }^{[7]}$ 构建高 新技术产业投入能力、产出能力、创新能力、产业支持 环境的评价指标体系,运用数据挖掘方法(K-均值聚类)进 行高新技术产业竞争力评价。张月花等（2017） ${ }^{[8]}$ 根据 高技术产业知识产权成果产出、技术研发水平、财政支 持、环境支撑四个层次构建指标体系，运用因子分析法 将陕西省与中国内地其它 30 个省市进行比较分析。何向 武和周文泳（2018） ${ }^{[9]}$ 根据产业生产经营水平、研发创 新活动水平和固定资产投资水平三者协同演化提出了一 套较为系统的区域高技术产业创新生态系统协同性的评 价体系。郭建平（2018） ${ }^{[10]}$ 选用数据包络分析方法, 将 高新技术企业就业人员、科技活动人员、科技活动经费 作为投入变量, 高新技术企业经济效益、社会效益作为 产出变量, 构建高新技术企业投入产出效率评价指标体 系。除了上述提到的评价方法, 还包括以下几种：模糊 评价模型 ${ }^{[11]}$ 、主成分分析 ${ }^{[12]} 、$ DEA-Malmquist 指数方法

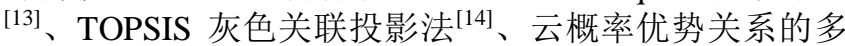
准则决策方法 ${ }^{[15]}$ 等。

通过以上的学者研究可知, 产业评价指标体系和评 价方法在不断地进行发展和完善。然而, 就评价方法而 言, 方法过于单一化, 组合运用的方法较少。因而, 本 文引入犹豫模糊语言 TOPSIS 方法, 首先运用熵权法计 算指标权重，接着在犹豫模糊语言的基础上，将犹豫模 糊语言和 TOPSIS 多属性决策的方法相结合。通过犹豫 模糊语言得到的评价值增强了决策者信息表达的可信性 和灵活度, 再使用 TOPSIS 法计算出方案与正负理想解 的距离并进行排序, 因为并没有主观的添加隶属值, 因 而保证了评价结果的客观性、真实性和精准性。相比于 单一的评价方法，犹豫模糊语言 TOPSIS 法更具有合理 性和科学性。

就评价指标体系来讲, 现有的产业评价指标体系缺 少对互联大数据的运用，可能会导致信息缺失，并且大 数据产业作为国家的数字性发展战略, 需要互联网大数 据的具体指标作为支撑。近几年来, 搜索引擎数据已经 成为国内外学者们研究的一大热点。国外学者中, Johnson (2004) ${ }^{[16]}$ 和谷歌工程师 Ginsberg (2009) ${ }^{[17]}$ 将 其运用到医学; Amuri 和 Marcucci (2012) [18] 运用搜索 引擎数据失业率; Choi 和 Varian (2012) ${ }^{[19]}$ 研究如何利 用搜索引擎数据预测短期经济价值; Da、 Engelberg 和 Gao（2015） ${ }^{[20]}$ 依据百万户民众的互联网日搜索数据揭 示市场情绪的变化。在国内研究中, 俞庆进和张兵 (2012) ${ }^{[21]}$ 采用百度指数作为投资者关注的代理变量, 考察创业板股票市场的波动; 董倩、孙娜娜和李伟 (2014) ${ }^{[22]}$ 以百度搜索指数为数据基础, 采用多种计量 模型对二手房和新房价格进行拟合和预测; 缪杰 (2014) ${ }^{[23]}$ 利用百度指数所提供的关键词搜索量数据, 研究了投资者关注度对公司股票市场表现的影响; 孟雪 井等 (2016) ${ }^{[24]}$ 利用文本挖掘技术构建了三大词库，利 用因子分析方法构建了具有先行预测作用的沪市投资者 情绪指数; 陈声利等（2018） ${ }^{[25]}$ 首次将百度指数引入 HAR 波动建模框架, 基于跳跃、好坏波动率与百度指数 提出 HAR 改进模型, 实证研究揭示股指期货波动运行 规律。所以，本文创新性的提出对搜索引擎数据的应用 来研究大数据产业的发展现状, 即通过百度指数这一指 标来反映大数据产业的影响力, 从而进行省域大数据产 业发展水平评价。
通过上述文献了解到, 现有的评价指标体系存在不 完全性, 评价方法存在一定的单一性。鉴于此, 本文在 引入百度指数作为搜索引擎数据来体现大数据产业的影 响力的基础上, 采用犹豫模糊语言 TOPSIS 方法对全国 的大数据产业的发展水平进行评价。具体结构安排如 下: 首先, 介绍基于犹豫模糊语言 TOPSIS 方法; 接 着, 介绍选取的指标体系以及通过实证来分析贵州大数 据产业发展水平; 最后, 运用犹豫模糊语言 TOPSIS 法 对大数据产业发展水平进行实证测评, 进而提出相关结 论。

\section{I. 地基于犹豫模糊语言的 TOPSIS 方法介绍}

\section{A. 犹豫模糊语言的预备知识}

在多属性决策问题中，由于现实世界的复杂性和 人们认识的不全面性及模糊性, 决策者很难对具体的属 性作出准确的数值评价。相比于给出精准的数值评价, 决策者更愿意用语言属于进行方案评价。犹豫模糊语言 集是指语言变量的取值为语言术语集上的一个有序且连 贯的子集。同时文本自由语法提供了将语言表达术语与 模糊语言集相对应的语法规则, 通过文本自由语法就可 以将语言表达式转化为能够运算的犹豫模糊语言集。

定义 $1:$ 设 $\mathrm{S}=\left\{s_{\alpha} \mid \alpha=-\tau, \ldots,-1,0,1, \ldots, \tau\right\}$ 为一个 语言术语集, $\tau$ 为正整数。若 $H_{s}$ 是 $\mathrm{S}$ 中有限个有序的连 续语言术语的集合, 则称 $H_{s}$ 为 $\mathrm{S}$ 上的一个犹豫模糊语言 术语。 $x_{i} \in X, i=1,2, \ldots, N$, 则犹豫模糊语言集的数学 形式为:

$$
H_{s}=\left\{<x_{i}, h_{s}\left(x_{i}\right)>\mid x_{i} \in X\right\}
$$

其中, 函数 $H_{s}: \mathrm{X} \rightarrow \mathrm{S}$ 表示元素 $x_{i} \in X$ 映射到 $\mathrm{A} \subset \mathrm{X}$ 的可能隶属度, $h_{s}\left(x_{i}\right)$ 是语言术语集 $\mathrm{S}$ 中的可能取值, 且 $h_{s}\left(x_{i}\right)=\left\{s_{\delta_{l}}\left(x_{i}\right) \mid s_{\delta_{l}}\left(x_{i}\right) \epsilon S, l=1,2, \ldots, \# \mathrm{H}_{\mathrm{s}}\right\}$ $\delta_{l} \in\{-\tau, \ldots,-1,0,1, \ldots, \tau\}$ 为语言术语 $s_{\delta_{l}}\left(x_{i}\right)$ 的下标, $\# \mathrm{H}_{\mathrm{s}}$ 为 $h_{s}\left(x_{i}\right)$ 中语言术语的个数。这种下表对称的语言 术语集 $\mathrm{S}$ 满足以下的条件:

（1）有序性: 若 $\mathrm{i}>\mathrm{j}$ 时, 则 $s_{;}>s_{j}$;

(2) 存在负算子: $\operatorname{Neg}\left(s_{\alpha}\right)=s_{-\alpha}$, 特别地, $\operatorname{Neg}\left(s_{0}\right)=s_{0}$

例如: 当 $\tau=3$ 时, 含有七个语言术语的语言标度 $S$ 为

$\mathrm{S}=\left\{s_{-3}=\right.$ 极差, $s_{-2}=$ 很差, $s_{-1}=$ 差, $\mathrm{s}_{0}=$ 中等,

$s_{1}=$ 好, $s_{2}=$ 很好, $s_{3}=$ 极好 $\}$

定义 2: 在决策的过程中, 为了便于运算, 又将离散 的术语犹豫模糊语言集推广到了连续型的犹豫模糊语言 集 $\bar{S}=\left\{S_{\alpha} \mid \alpha \epsilon[-q, q]\right\}$ ，其中 $q$ 是一个足够大的正数。 $\bar{S}=\left\{\overline{S_{\alpha}} \in S\right\}$ 作为一种虚拟语言术语只在计算中出现, 满 足上边 1) 和 2)。若 $s_{\alpha}, s_{\beta} \in \bar{S}, \gamma \in[0,1]$, 则满足以下条 件:

(1) $s_{\alpha} \oplus s_{\beta}=s_{\alpha+\beta}, s_{\alpha}-s_{\beta}=s_{\alpha} \oplus \operatorname{Neg}\left(s_{\beta}\right)$;

(2) $\gamma s_{\alpha}=s_{\gamma \alpha}$ 
定义 3 : 设 $E_{G_{H}}$ 为将文本自由语法 $G_{H}$ 生成的语言表 达式ll $\in S_{l l}$ 转化为犹豫模糊语言集 $H_{s}$ 的函数, $\mathrm{S}$ 为语法 $G_{H}$ 所采用的语言术语集, $S_{l}$ 为语法 $G_{H}$ 的生成规则所生 成的语言表达式的集合, 则由语法 $G_{H}$ 所生成的语言表达 式可通过转化公式 $E_{G_{H}}: S_{l l} \rightarrow H_{s}$ 转换成犹豫模糊语言 集, 其中

$$
\begin{aligned}
& E_{G_{H}}\left(s_{g}\right)=\left\{s_{g} \mid s_{g} \in S\right\} ; \\
& E_{G_{H}}\left(\text { 至多 } s_{\alpha}\right)=\left\{s_{g} \mid s_{g} \in S, \text { 且 } s_{g} \leq s_{\alpha}\right\} ; \\
& E_{G_{H}}\left(\text { 少于 } s_{\alpha}\right)=\left\{s_{g} \mid s_{g} \in S, \text { 且 } s_{g}<s_{\alpha}\right\} ; \\
& E_{G_{H}}\left(\text { 至少 } s_{\alpha}\right)=\left\{s_{g} \mid s_{g} \in S, \text { 且 } s_{g} \geq s_{\alpha}\right\} ; \\
& E_{G_{H}}\left(\text { 多于 } s_{\alpha}\right)=\left\{s_{g} \mid s_{g} \in S, \text { 且 } s_{g}>s_{\alpha}\right\} ; \\
& E_{G_{H}}\left(\text { 在 } s_{\alpha} \text { 和 } s_{\beta} \text { 之间 }\right)= \\
& \left\{s_{g} \mid s_{g} \in S, \text { 且 } s_{\alpha} \leq s_{g} \leq s_{\beta}\right\}
\end{aligned}
$$

B. 犹豫模糊语言 TOPSIS 方法计算步骤

设 $\mathrm{X}=\left\{x_{1}, x_{2}, \ldots, x_{n}\right\}$ 为方案集 $\mathrm{C}=\left\{c_{1}, c_{2}, \ldots, c_{m}\right\}$ 的属 性集, $\mathrm{W}=\left\{w_{1}, w_{2}, \ldots w_{n}\right\}$ 是属性的权重向量, 满足 $\mathrm{w}_{j} \in[0,1]$, 且 $\sum_{j}^{n} \mathrm{w}_{j}=1$ 。语 言 标度 $\mathrm{S}=\left\{s_{\alpha} \mid \alpha=-\tau, \ldots,-1,0,1, \ldots, \tau\right\}$, 各专家在此标度下给 出方案 $x_{i}$ 在属性 $c_{j}$ 下的评估, 并通过 $E_{G_{H}}$ 转化为犹豫模 糊语言集。通过决策对方案进行排序, 对方案进行比 较。

基于犹豫模糊语言的 TOPSIS 法的思想, 建立犹豫 模糊多属性决策方法, 具体的步骤如下:

步骤 1: 通过文本自由法将语言信息转化成犹豫模 糊语言集, 得到犹豫模糊语言决策矩阵 $\mathrm{R}=\left(H_{S}^{i j}\right)_{n \times m}$, 其中 $H_{S}^{i j}$ 表示专家对方案 $x_{i}$ 在属性 $c_{j}$ 下的语言评价值。因 此犹豫模糊语言决策矩阵可表示成如下:

$$
\mathrm{R}=\left(H_{S}^{i j}\right)_{n \times m}=\left\{\begin{array}{cccc}
H_{S}^{11}\left(x_{1}\right) & H_{S}^{12}\left(x_{1}\right) & \ldots & H_{S}^{1 n}\left(x_{1}\right) \\
H_{S}^{21}\left(x_{2}\right) & H_{S}^{22}\left(x_{2}\right) & \ldots & H_{S}^{2 n}\left(x_{2}\right) \\
\vdots & \vdots & \ddots & \vdots \\
H_{S}^{m 1}\left(x_{m}\right) & H_{S}^{m 1}\left(x_{m}\right) & \ldots & H_{S}^{m n}\left(x_{m}\right)
\end{array}\right\}
$$

对于不同的 $H_{S}^{i j}$, 可能包含的语言术语的个数是不相 同的, 我们选择添加某虚拟语言术语到较少 $H_{S}^{i j}$ 之中, 使 得不同的 $H_{S}^{i j}$ 所包含的语言术语的个数是相同的。设 $\mathrm{b}=\left\{b_{l} \mid l=1,2, \ldots, \# b\right\}$ 为犹豫模糊语言数, \# $b$ 为 $b$ 中 语言术语的个数, 令 $b^{+}$和 $b^{-}$为 $b$ 中的最大和最小语言术 语, $\xi(0 \leq \xi \leq 1)$ 为最优参数, 则可添加语言术语 $\mathbf{b}=\xi b^{+} \oplus(1-\xi) b^{-}$至个数较少的犹豫模糊语言中。 $\xi$ 的取值取决于决策者对风险的态度。乐观的决策者会选 择添加最大的虚拟语言术语, 即 $\xi=1$ 。悲观的决策者则 选择增加最小的虚拟语言术语, 即 $\xi=0$ 。中立型的决策
者则会取 $\xi=\frac{1}{2}$ 。因为决策者对风险的态度不一致最终决 策结果也将不相同。本文中我们取 $\xi=\frac{1}{2}$ 。

$$
\text { 例 如 : } H_{S}^{11}=\left\{s_{1}, s_{2}\right\} \quad, \quad H_{S}^{12}=\left\{s_{1}\right\} \text {, }
$$
$H_{S}^{13}=\left\{s_{1}, s_{2}, s_{3}\right\}$, 则对于 $H_{S}^{11}$ 我们添加 $s_{1.5}=\frac{s_{1} \oplus s_{2}}{2}$ 到 $_{S_{S}^{11}}^{11}$ 中, 即得到 $H_{S}^{11}=\left\{s_{1}, s_{1.5}, s_{2}\right\}$, 同理 $H_{S}^{12}=\left\{s_{1}, s_{1}, s_{1}\right\}$ 。

步骤 2: 得到犹豫模糊语言的正负理想解。正理想 解 $A^{+}=\left\{H_{s}^{1+}, H_{s}^{2+}, \ldots, H_{s}^{n+}\right\}$, 负 理 想 解 $A^{-}=\left\{H_{s}^{1-}, H_{s}^{2-}, \ldots, H_{s}^{n-}\right\}$, 其中对于效益型的指标而言 正理想解是由每一列最大的一个犹豫模糊语言术语组 成, 负理想解是由每一列最小的一个犹豫模糊语言术语 组成。而对于成本型指标而言, 正理想解是由每一列最 小的一个犹豫模糊语言术语组成, 负理想解是由每一列 最大的一个犹豫模糊语言术语组成。

步骤 3: 计算方案 $x_{i}$ 与正、负理想解之间的加权距离 $D_{i}^{+}$和 $D_{i}^{-}$。在计算加权距离之前我们先来了解一下两个 犹豫模糊语言集之间的欧式距离。设扩展标度 $\bar{S}=\left\{S_{i} \mid i \epsilon[-q, q]\right\} \quad, \quad H_{S}^{11}=\left\{b_{1}^{11}, b_{2}^{11}, \ldots, b_{l}^{11}\right\} \quad$ 和 $H_{S}^{22}=\left\{b_{1}^{22}, b_{2}^{22}, \ldots, b_{l}^{22}\right\}$, 记 $\delta($.$) 为虚拟语言术语的下$ 标。令

$$
\mathrm{d}\left(H_{S}^{11}, H_{S}^{22}\right)=\left(\frac{1}{l} \sum_{i=1}^{l}\left(\frac{\delta\left(b_{i}^{22}\right)-\delta\left(b_{i}^{22}\right)}{2 q}\right)^{2}\right)^{\frac{1}{2}}
$$

为标准的欧式距离。那么利用欧式距离公式计算的 方案 $x_{i}$ 与正、负理想解之间的加权距离 $D_{i}^{+}$和 $D_{i}^{-}$为:

$$
\begin{aligned}
& D_{i}^{+}=\sum_{j=1}^{m} w_{j} d\left(H_{S}^{i j}, H_{s}^{j+}\right) \\
& D_{i}^{-}=\sum_{j=1}^{m} w_{j} d\left(H_{S}^{i j}, H_{s}^{j-}\right)
\end{aligned}
$$

步骤 4: 各方案 $x_{i}$ 与理想解的相对贴近度 $R C_{i}$

$$
R C_{i}=\frac{D_{i}^{-}}{D_{i}^{+}+D_{i}^{-}}, \quad \mathrm{i}=1,2, \ldots, \mathrm{n}
$$

其中相对贴近度越大代表着方案 $x_{i}$ 越优, 根据相对 贴近度的大小对方案 $x_{i}$ 进行排序。

\section{II. 实证分析}

\section{A.评价指标体系构建}

结合大数据产业的发展的实际情况, 本研究以科学 性、系统性、全面性、可获得性为原则, 把大数据产业 发展的指标体系分为目标层、准则层和因素层。其中基 准层包括大数据产业的产业规模、产品类型、招商引 资、基础设施以及产业影响力 (百度指数) 。指标层共 有 29 个具体的指标来反映大数据产业的实际发展水平, 且 29 个指标均为效益型指标。如此, 建立大数据产业发 展水平的指标体系, 如表 1 所示。

电子信息产业为大数据产业发展提供产业基础,在反 映大数据产业的发展水平上具有一定的代表性。同时, 以百度指数为代表的搜索引擎指数来反映大数据产业的 影响力。因而, 本文研究数据主要来源于中国电子信息 产业统计年鉴（2013-2017）、中国统计局中运输和邮电 
表 1 大数据产业发展水平评价体系及权重

\begin{tabular}{|c|c|c|c|}
\hline 目标层 & 准则层 & 因素层 & 权重 \\
\hline \multirow[t]{29}{*}{ 大数据产业 } & \multirow[t]{11}{*}{ 产业规模 } & 规模以上企业个数 ( 个 ) & 0.036 \\
\hline & & 主营业务收入 ( 亿元 ) & 0.037 \\
\hline & & 利润总额 ( 亿元 ) & 0.036 \\
\hline & & 主营业务成本 ( 亿元 ) & 0.036 \\
\hline & & 资产总计 ( 亿元 ) & 0.034 \\
\hline & & 负债合计 ( 亿元 ) & 0.034 \\
\hline & & 本年累计完成投资 ( 亿元 ) & 0.020 \\
\hline & & 本年新增固定资产 ( 亿元 ) & 0.023 \\
\hline & & 施工项目个数 ( 个 ) & 0.022 \\
\hline & & 出口完成 ( 万美元 ) & 0.049 \\
\hline & & 进口情况 ( 万美元 ) & 0.048 \\
\hline & \multirow[t]{8}{*}{ 产品类型 } & 通信设备 ( 万美元 ) & 0.057 \\
\hline & & 广播电视设备 ( 万美元 ) & 0.067 \\
\hline & & 计算机 ( 万美元 ) & 0.053 \\
\hline & & 家用电子电器 ( 万美元 ) & 0.062 \\
\hline & & 电子元件 ( 万美元 ) & 0.060 \\
\hline & & 电子器件 ( 万美元 ) & 0.049 \\
\hline & & 电子材料 ( 万美元 ) & 0.026 \\
\hline & & 电子仪器设备 ( 万美元 ) & 0.063 \\
\hline & \multirow[t]{3}{*}{ 招商引资 } & 国家预算内资金 ( 亿元 ) & 0.039 \\
\hline & & 国内贷款 ( 亿元 ) & 0.027 \\
\hline & & 利用外资（亿元） & 0.059 \\
\hline & \multirow[t]{5}{*}{ 基础设施 } & 移动电话基站 & 0.009 \\
\hline & & 互联网宽带接入用户数 (万户 ) & 0.011 \\
\hline & & 移动电话交换机容量 ( 万户 ) & 0.008 \\
\hline & & 长途光缆线路长度 ( 公里) & 0.008 \\
\hline & & 互联网宽带接入端口 ( 万个 ) & 0.010 \\
\hline & \multirow{2}{*}{$\begin{array}{c}\text { 产业影响力（百 } \\
\text { 度指数） }\end{array}$} & 搜索指数 PC 端 (频次 ) & 0.010 \\
\hline & & 搜索指数移动端 (频次 ) & 0.007 \\
\hline
\end{tabular}

注: 权重的数值得计算步骤详见 3.3

\section{B. 数据来源}

行业的电信主要通信能力（2013-2017）以及百度指数中 的贵州省 “大数据” 名词搜索指数（2013-2017），因为 没有 “大数据产业” 这一关键词的搜索指数, 所以我们 用 “大数据” 的搜索指数进行代替。个别缺失的数据通 过一系列的估算方法得到。因为文章的篇幅有限, 所以 原始数据不在此列出。

\section{C.指标权重的确定}

为了比较客观的对各项指标进行赋权, 本研究采用 熵权法来计算指标权重。熵权法就是指通过大数据产业 的发展指标的差异程度的大小, 来反映指标的权重, 使 得各指标的权重更加精准。具体的计算步骤如下（结果 如表 1 所示）:
假设对 $\mathrm{m}$ 个评价对象 $\mathrm{n}$ 个评价指标进行贵州各地级 市大数据产业发展水平的评价首先建立如下的原始数据 矩阵:

$$
\mathrm{R}=\left[\begin{array}{cccc}
r_{11} & r_{12} & \ldots & r_{1 \mathrm{n}} \\
r_{21} & r_{22} & \ldots & r_{2 n} \\
\vdots & \vdots & \vdots & \ldots \\
r_{m 1} & r_{m 2} & \ldots & r_{m n}
\end{array}\right]
$$

(1) 对原始数据矩阵进行标准化处理得到: $X_{i j}=\left(x_{i j}\right)_{m n}$ 
$x_{i j}$ 为第 $\mathrm{i}$ 个评价对象在第 $\mathrm{j}$ 个评价指标上的标准 值, $x_{i j} \in[0,1]$ 。其中对大者为优的收益性指标而 言, 有:

$$
x_{i j}=\frac{r_{i j}-\min _{j} r_{i j}}{\max _{i} r_{i j}-\min _{i} r_{i j}}
$$

对于小者为优的指标则有:

$$
x_{i j}=\frac{\max _{j} r_{i j}-r_{i j}}{\max _{i} r_{i j}-\operatorname{minr}_{i j}}
$$

其中 $\max _{j} r_{i j}$ 及 $\min _{j} r_{i j}$ 分别为 $\mathrm{j}$ 个评价指标下所有评价 对象的原始数值的最大值及最小值。

(2) 将 $x_{i j}$ 转化成 $p_{i j}$ :

$$
p_{i j}=\frac{x_{i j}}{\sum_{i=1}^{m} x_{i j}} \mathrm{i}=1,2 \ldots, \mathrm{m} \mathrm{j}=1,2 \ldots, \mathrm{n}
$$

（3）定义第 $\mathrm{j}$ 个指标的熵为

$$
\mathrm{H}_{j}=-\mathrm{k} \sum_{i=1}^{m} p_{i j} \ln p_{i j}, \quad \mathrm{j}=1,2 \ldots, \mathrm{m},
$$

其中 $\mathrm{k}=\frac{1}{\ln m}$ 。

（4）定义第 $\mathrm{j}$ 个指标的熵权为:

$$
\mathrm{w}_{j}=\frac{1-H_{j}}{\sum_{j=1}^{n}\left(1-H_{j}\right)}=\frac{1-H_{j}}{n-\sum_{j=1}^{n} H_{j}}, j=1,2 \ldots, n,
$$

其中 $\mathrm{w}_{j} \in[0,1]$, 且 $\sum_{j}^{n} \mathrm{w}_{j}=1$ 。

根据全国 31 省的数据我们可以得到 2013-2017 每年 的 29 个指标属性的权重, 为了更好的进行分析我们将每 个属性五年的权重加总再求平均, 从而统一权重值, 得 到表 1 中的最终属性权重值。

\section{D. 实证结果}

由实际得到的以及估算数据我们已经求出 29 个指标 各自所占的权重。现在我们决定采用 $\mathrm{S}=\left\{s_{-3}=\right.$ 极差, $s_{-2}=$ 很差, $s_{-1}=$ 差, $\mathrm{s}_{0}=$ 中等, $\mathrm{s}_{1}=$ 好, $\mathrm{s}_{2}=$ 很好, $\mathrm{s}_{3}=$ 极好 $\}$

这七个语言术语标度让专家根据所得的数据进行语言评 价, 从而得到犹豫模糊语言决策矩阵、正负理想值以及

\begin{tabular}{|c|c|c|c|c|c|c|}
\hline & 2013 年 & 2014 年 & 2015 年 & 2016 年 & 2017 年 & 平均值 \\
\hline 北京 & & 0.070326 & 0.036531 & 0.040755 & 0.043013 & 0.044353 \\
\hline 天津 & 0.030593 & 0.040732 & 0.033974 & 0.018526 & 0.012181 & 0.027201 \\
\hline 河北 & 0.021167 & 0.024978 & 0.027889 & 0.026162 & 0.056299 & 0.031299 \\
\hline 山西 & 0.010429 & 0.038488 & 0.012321 & 0.014178 & 0.018076 & 0.018698 \\
\hline 内蒙古 & 0.01125 & 0.022091 & 0.029386 & 0.025307 & 0.029167 & 0.02344 \\
\hline 辽宁 & 0.019138 & 0.020875 & 0.029464 & 0.032373 & 0.055404 & 0.031451 \\
\hline 吉林 & 0.00705 & 0.009335 & 0.016381 & 0.009304 & 0.015589 & 0.011532 \\
\hline 黑龙江 & 0.010566 & 0.013523 & 0.015465 & 0.017448 & 0.01879 & 0.015159 \\
\hline 上海 & 0.149761 & 0.173929 & 0.146431 & 0.166696 & $0.1^{\prime}$ & 0.16204 \\
\hline 江苏 & 0.424583 & 0.442181 & 0.4566 & & & 0.46054 \\
\hline 浙江 & 0.096478 & 0.14 & & & & 0.144539 \\
\hline 安徽 & 0.034456 & 0.03 & & & & 3235 \\
\hline 福建 & 0.061643 & 0.06 & 0.05 & & & 0.069899 \\
\hline 江西 & & & & & & \\
\hline 山东 & & & & & & \\
\hline 河南 & & $0.0^{\prime}$ & & & & 0.09 \\
\hline 湖北 & & & 0.04 & & & 4682 \\
\hline 湖南 & & 0.05 & & & & 0.048991 \\
\hline 广东 & 0.565189 & 0.569546 & 0.672323 & 0.67 & 0.81 & 0.658401 \\
\hline 广西 & 0.012828 & 0.016243 & 0.024505 & & & 0.024712 \\
\hline 海南 & 0 & & & & & \\
\hline 重) & & & & & & 833 \\
\hline 四川 & & & & & & 948 \\
\hline 贵州 & & & & & & \\
\hline & & & & & & \\
\hline & & & & & & 184 \\
\hline 陕西 & & & & & & 344 \\
\hline 甘肃 & & & & & & 0.009879 \\
\hline & & & & & & 0.005948 \\
\hline 宁夏 & & & & & & 0.003781 \\
\hline 新疆 & 0.011908 & 0.010386 & 0.010188 & 0.01277 & 0.015881 & 0.012226 \\
\hline
\end{tabular}
欧式距离, 由于文章篇幅的原因就不再一一列出。最后 得到 2013-2015 年的相对贴近度以及平均值, 如下表 2 所示。

\section{表 2 全国各省市的相对贴进度及五年平均值}


从表 2 的相对贴近度的平均值我们可以得到, 全国 三十一个省份大数据产业的发展水平整体上不断提升, 广东、江苏的大数据产业发展水平排名位于前列, 且大 数据发展水平远超其他省份; 上海、浙江、山东大数据 发展水平位列全国第三、第四、第五，且相对贴进度均 超过 1 ; 西藏、青海、海南、宁夏排名处于末端, 即大 数据发展水平比较落后。全国不同省市之间差距比较 大，增速也各有不同，但从整体上可知我国大数据产业 正处于不断发展的阶段。

\section{III. 结论与讨论}

为了准确的评价近五年三十一个省市大数据产业发展 的水平，本文首先选取了大数据产业的产业规模、产品 类型、招商引资、基础设施以及百度指数这五个指标来 详细、具体的反映大数据产业的实际发展情况; 其次采 用熵权法比较客观的计算 29 个属性指标的具体权重; 最 后通过采用基于犹豫模糊语言的 TOPSIS 方法, 尽量去 克服客观世界的复杂性和人们认识的不全面性得到比较 符合认知、更可行可信的大数据产业发展水平的评价结 果。通过实证结果可得到以下结论:

1）时间上，从 2013 年到 2017 年各省大数据产业发 展水平均呈现不断发展的态势。其中，广西、辽宁、内 蒙古的增长率位于全国前三，大数据产业发展在样本期 间内有明显的发展; 而天津、四川、青海相对贴进度 2017 年比 2013 年增长率为负, 即表明这三个省份的大 数据产业发展并不稳定, 且有退后趋势

2）空间上, 广东省的大数据产业发展水平最高, 其 次是江苏省，且两省的大数据产业发展水平远远超过其 他省份; 而西藏、青海、海南、宁夏处于比较低的排 名, 即大数据产业的发展水平较为落后。样本期间内, 大数据产业发展水平呈省域不均衡。

\section{致谢}

本项目受国家自然科学基金地区项目 (71861003) 和 2017 年度第二批贵州省基础研究计划（软科学类别）项 目（黔科合基础（2017）1516-1）联合资助。

\section{参考文献}

[1] 工业和信息化部.大数据产业发展规划(2016-2020 年)(工信部规 (2016) 412 号)[EB/OL]. http://www.miit.gov.cn,2017-1-17

[2] 唐中赋,顾培亮.高新技术产业发展水平的综合评价. 经济理论与经 济管理,2003(10):23-28.

[3] 任伟宏,黄鲁成.基于熵的研发产业发展水平评价模型及应用.科技 进步与对策,2009,26(03):109-111.

[4] 李晓鸿.陕西电子信息产业循环经济发展评价指标体系与评价方 法研究.科技管理研究,2010,30(03):64-66+72.

[5] 王海龙,刘佳.我国省区高技术产业发展水平评价与模式分析.科技 进步与对策,2011,28(22):113-117.

[6] 周叶.江西高新技术产业发展水平分析一一基于多因子等权评价 模型.江西社会科学,2012,32(10):253-256.

[7] 李刚.我国高技术产业发展水平与集群水平评价及相关关系研究. 工业技术经济,2014,33(01):29-33。

[8] 张镧.湖北省高新技术开发区产业发展水平的评价. 统计与决 策,2015(03):55-57.

[9] 桂俊爆.我国省域高技术产业发展水平测度与提升策略.经济纵 横,2018(07):83-92
[10] 俞庆进, 张兵. 投资者有限关注与股票收益一一以百度指数作为关 注度的一项实证研究.金融研究,2012(08):152-165.

[11] 董倩,孙娜娜,李伟.基于网络搜索数据的房地产价格预测. 统计研 究, 2014,31(10):81-88.

[12] 缪杰. 基于百度指数的投资者关注度对于股票市场表现的影响 $[\mathrm{D}]$. 厦门大学, 2014 .

[13] 孟雪井,孟祥兰,胡杨洋.基于文本挖掘和百度指数的投资者情绪指 数研究.宏观经济研究,2016(01):144-153.

[14] 徐映梅, 高一铭. 基于互联网大数据的 CPI 與情指数构建与应用一 一以百度指数为例.数量经济技术经济研究, 2017,34(01):94-112.

[15] 陈声利.关涛, 李一军. 基于跳跃、好坏波动率与百度指数的股指期 货波动率预测.系统工程理论与实践,2018,38(02):299-316.

[16] Rodrı 'guez R M, Martı nez L, Herrera F. Hesitant fuzzylinguistic terms sets for decision making. IEEE Transactions on Fuzzy Systems, 2012, 20: 109-119.

[17] Liu H B, Rodriguez R M. A fuzzy envelope of hesitant fuzzy linguistic term set and its application to multicriteria decision making Information Sciences, 2014, 258: 220-238.

[18] Wei C P, Zhao N, Tang X J. Operators and comparisons of hesitant fuzzy linguistic term sets. IEEE Transactions on Fuzzy Systems, 2014, 22(3): 575-585

[19] Liao H C, Xu Z S, Zeng X J, et al. Qualitative decision making with correlation coefficients of hesitant fuzzy linguistic term set. Knowledge-Based Systems, 2015, 76:127-138.

[20] 邹志红,孙靖南,任广平. 模糊评价因子的熵权法赋权及其在水质评 价中的应用.环境科学学报,2005(04):552-556.

[21] 王否, 周亚楠,张宇.基于熵权-TOPSIS 法的低碳城市发展水平评价 及障碍度分析-—以天津市为例. 科技管理研究, 2017,37(17):239245

[22] 张目,周宗放.基于粗集和熵权-TOPSIS 法的高新技术企业自主创 新能力评价.数学的实践与认识,2008,38(24):52-58.

[23] 廖虎昌,杨竹,徐泽水,顾新.犹豫模糊语言 PROMETHEE 方法在川 酒品牌评价中应用 [J/OL].控制与决策:1-10[2019-04-13].

[24] 魏翠萍,葛淑娜. 犹豫模糊语言幕均算子及其在群决策中的应用.系 统科学与数学, $2016,36(08): 1308-1317$ 